\title{
Trajectories of microwave prominence eruptions
}

\author{
K. Hori` and J. L. Culhane \\ Mullard Space Science Laboratory, University College London, Holmbury St. Mary, Dorking, Surrey, \\ RH5 6NT, UK
}

Received 15 March 2001 / Accepted 21 November 2001

\begin{abstract}
On the basis of $17 \mathrm{GHz}$ microwave images from the Nobeyama Radioheliograph near solar maximum (1999-2000), we examined trajectories of 50 prominence eruptions in order to address how prominence motions affect or reflect the surrounding coronal structures. We marked the heliocentric latitude of the top of the moving prominences on the white-light synoptic maps, which were constructed at 2.5-4.5 solar radii from the LASCO C2 coronagraph on the $S O H O$ spacecraft. We found that i) $92 \%$ of the prominence eruptions were associated with coronal mass ejections (CMEs) and that the remaining $8 \%$ show weak mass motions confined to nearby streamers, and ii) coronal mass motions involving prominence eruptions and CMEs are not random but are organized by bundles of streamers. Our findings support the idea of multiple plasma sheets emanating from active regions, arcades, trans-equatorial interconnecting loops, and polar crown filaments, through which coronal mass is transported toward interplanetary space. This study suggests that microwave observations can provide useful information on the activity at the base of such "coronal mass corridors".
\end{abstract}

Key words. Sun: prominences - Sun: CME - Sun: corona - Sun: activity

\section{Introduction}

It is well known that coronal mass is transported into interplanetary space in various forms of solar activity. Coronal mass ejections (CMEs) are the most spectacular mass transportation that are not necessarily associated with flares, i.e., the explosive energy dissipation process (up to $10^{32} \mathrm{erg}$ in $10^{2}$ to $10^{3} \mathrm{~s}$ ) in the lower atmosphere (e.g., Munro et al. 1979; Gosling 1997; Howard 1997). Previous studies have suggested that prominence eruptions are a common form of activity associated with the appearance of CMEs (Sheeley et al. 1975; Munro et al. 1979; Webb \& Hundhausen 1987; St Cyr \& Webb 1991; Hundhausen 1993). Hence, dynamic evolution of eruptive prominences has been highlighted as one of the keys to understanding the initiation of CMEs (e.g., Kahler et al. 1988; Wiik et al. 1997; Gilbert et al. 2000; Delannee et al. 2000). Recent white-light coronal images from the Large-Angle and Spectrometric Coronagraph (LASCO; Brueckner et al. 1995) on the Solar and Heliospheric Observatory $(S O H O)$ spacecraft have demonstrated that some helical prominences that erupted from the lower corona actually correspond to the bright (i.e., dense),

\footnotetext{
Send offprint requests to: K. Hori,

e-mail: Kuniko.Hori@msfc.nasa.gov

* Now at National Space Science and Technology Center, 320 Sparkman Drive, Huntsville, AL 35805, USA.
}

twisted CME core (e.g., Plunkett et al. 2000; Hori 2000; for a previous report, see Illing \& Hundhausen 1985). In the classical CME scenario (e.g., Low 1994) CMEs are associated with helmet streamer configurations: A quiescent prominence was formed over a magnetic neutral line within a streamer above, and then it becomes unstable and starts to erupt, resulting in a CME. Hori (2000) studied two microwave prominences that were initially not embedded in a streamer but moved toward the streamer latitude and then erupted or disappeared (Figs. 2c and 5c in Hori 2000). These prominences seem to be different from those in the classical CME scenario. Further, such prominence behavior suggests that streamers play the role of "mass corridor" through which coronal mass flows out toward the interplanetary space (Crooker et al. 1993).

Using the Solar Maximum Mission (SMM) coronagraph data, Hundhausen (1993) constructed white-light synoptic maps that provide coronal brightness (Thomsonscattered photospheric light) as a function of height over the solar limb and thus changes in the distribution of mass ejection latitudes can be examined. He found that about $50 \%$ of CMEs observed in 1984 were preceded by slow evolution of a bright, background corona at the mass ejection site and then produced a disruption of preexisting streamers (also see Jackson 1981; Sime 1989). On the other hand, Subramanian et al. (1999) have drawn different conclusions using LASCO data, which is superior to 


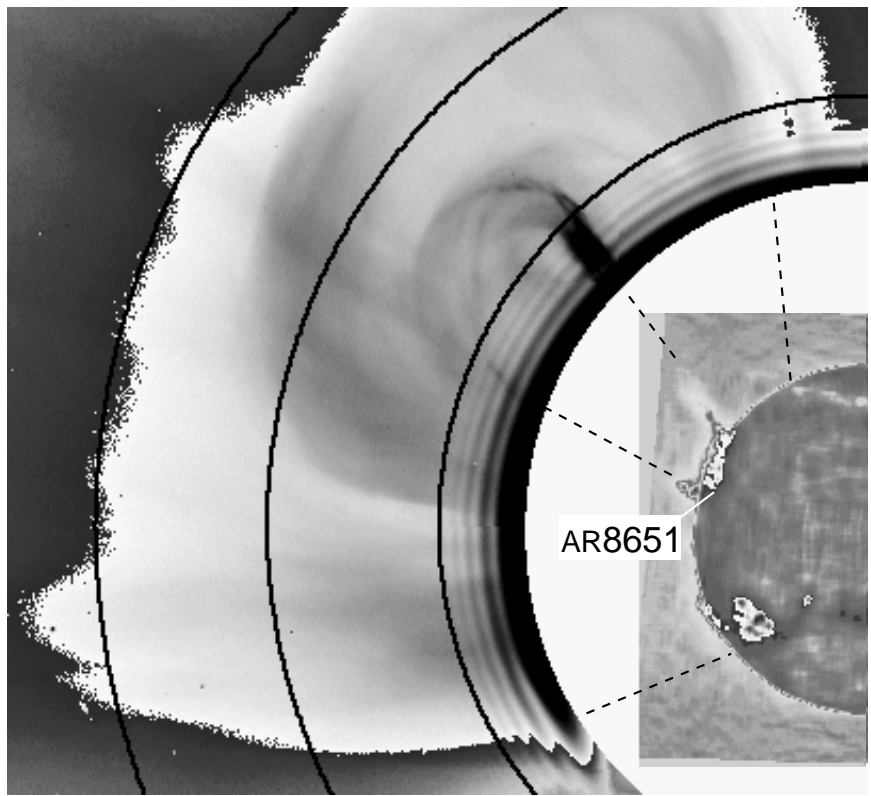

Fig. 1. The 27 July 1999 event. NoRH $17 \mathrm{GHz}$ (inner) and LASCO C2 (outer) images taken at 02:06 UT. From the inner, thick circles represent the location of $2.5,3.5$, and $4.5 R$ s from the Sun center. Solar north is up, and east is to the left.

SMM coronagraph data in both sensitivity and dynamic range. They reported that only $16 \%$ of the CMEs observed from January 1996 to June 1998 disrupted the preexisting streamers. Although $46 \%$ of CMEs showed an overlap with streamers, these seem to have had no effect on the streamers. They also found that $27 \%$ of all the CMEs are latitudinally displaced from the preexisting streamers and $8.5 \%$ create streamer-like structures. These reports again raise the question: Do CMEs really arise from preexisting helmet streamers?

In this paper, we investigate the trajectories of 50 prominence eruptions that were observed with the Nobeyama Radiohelograph (NoRH; Nakajima et al. 1994) at $17 \mathrm{GHz}$ and examine the relationship between prominence activity and the surrounding coronal structures. NoRH is a solar-dedicated radio interferometer that observes the Sun routinely for 8 hrs per day (independent of weather) with spatial and temporal resolution of $10^{\prime \prime}$ and $1 \mathrm{~s}$ at $17 \mathrm{GHz}$. Since NoRH detects the radio continuum emission, it can monitor the prominence activity over a wide range of velocities and temperatures. We combine the NoRH observations with the white-light synoptic maps from the LASCO C2 coronagraph and compare the latitudinal evolution of the coronal transients observed at different heights. This unique combination also enables us to further explore the origin of CMEs and their relation with the eruptive prominences.

In what follows, Sect. 2 describes our data and analysis method. Section 3 presents statistical features of the set of prominence activities studied, as well as a description of some characteristic events. In Sect. 4 a summary of the results is presented and their implications are discussed.

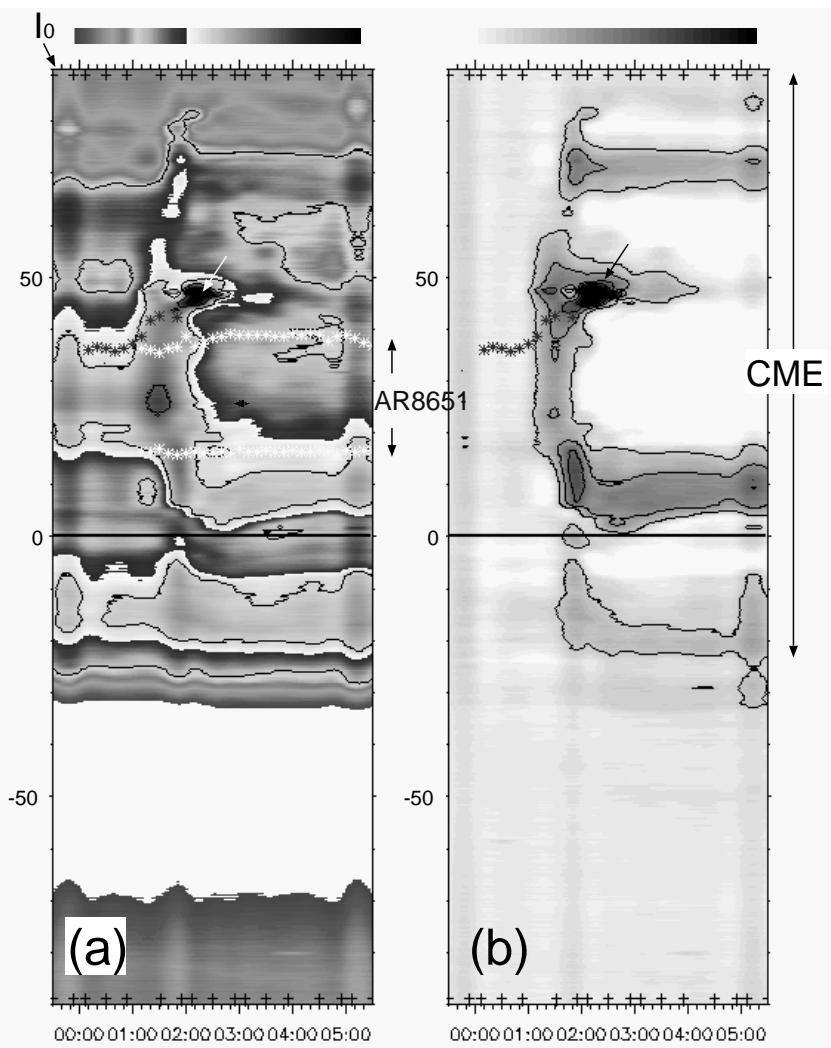

Fig. 2. East limb white-light synoptic maps constructed at $2.5 R$ s from the Sun center for the 27 July 1999 event. a) I map and b) $I-I_{0}$ map. A line of black asterisks shows the heliographic latitude of the top of the erupting prominence within $0.7 R \mathrm{~s}\left(5 \times 10^{5} \mathrm{~km}\right)$ from the solar surface; the upper limit of the NoRH field of view. A pair of lines of white asterisks shows the heliographic latitudes of the northern- and southern-ends of the AR 8651. Cross marks attached to the top and bottom of the maps show the times when the original C2 images were obtained. Horizontal axis shows time in UT. The contour levels are $25,50,75 \%$ of the maximum brightness of each synoptic map.

\section{Method}

While solar corona is optically thin and the chromosphere and quiescent prominences are optically thick in microwaves, the optical thickness of eruptive prominences can decrease at $17 \mathrm{GHz}$ as they expand (Fujiki 2000). For the optically thin free-free emission, the brightness temperature $\left(T_{\mathrm{b}}\right)$, which is the parameter that is provided by NoRH, can be written as

$T_{\mathrm{b}} \propto T^{-1 / 2} E M$

where $T$ and $E M$ stand for the temperature and emission measure of the source material. On the other hand,

$T_{\mathrm{b}} \sim T$,

for the optically thick emission if the filling factor is unity in the prominence. At $17 \mathrm{GHz}, T_{\mathrm{b}}$ for a quiescent prominence is a few $\times 10^{3} \mathrm{~K}$ lower than that of the quiet Sun, which is about $10^{4} \mathrm{~K}$. Hence, a prominence on the disk 
Table 1. $17 \mathrm{GHz}$ eruption list.

\begin{tabular}{|c|c|c|c|c|c|c|c|}
\hline No. Date & Location & Source $^{\mathrm{a}}$ & Time (UT) & $\operatorname{CME}(\mathrm{s})^{\mathrm{b}}$ & I & II III & Flares $^{\mathrm{c}} \&$ metric radio bursts \\
\hline 1. $2000-01-04$ & N37W50 & AR8810 & 03:02-03:41 & $03: 30-$ & $y$ & $y$ & 03:10-03:56-05:30 B9.2 \\
\hline 2. $2000-01-19$ & S39W90 & filament? & 04:50-06:00\# & $06: 54-$ & $y$ & $y$ & \\
\hline 3. $2000-02-09$ & N35W90 & AR8851? & $23: 10-01: 30$ & - & $y$ & $-\quad-$ & \\
\hline 4. $2000-02-12$ & N62E90+ & filament & $00: 30-04: 30$ & 21:08- NW, 04:31- & $n$ & $n$ & Type II, IV \\
\hline 5. $2000-02-16$ & S11W90 & AR8871? & $02: 30-03: 20$ & 01:00-, 03:30- NW & $y$ & $n \quad b$ & \\
\hline 6. $2000-02-17$ & N08W90 & QR? & $23: 30-02: 00$ & - & $y$ & $-\quad-$ & \\
\hline 7. $2000-02-22$ & N21W90 & AR8870? & $03: 20-04: 00$ & 01:31- SW, 03:54- & $n$ & $n \quad b / c$ & \\
\hline 8. $2000-02-25$ & N80W90 & QR & 01:00-06:11\# & $05: 30-$ & $y$ & $\begin{array}{ll}y & b\end{array}$ & \\
\hline 9. & $\mathrm{~N} 22 \mathrm{~W} 27$ & AR8879 & $05: 00-05: 40$ & $05: 30-$ & $y$ & $n \quad b$ & C flares \\
\hline 10. $2000-02-26$ & N22E51 & AR8889 & $23: 30-02: 00$ & $23: 54-$ & $y$ & $\begin{array}{ll}y & b\end{array}$ & \\
\hline 11. 2000-03-06 & S60W90 & QR? & $03: 40-04: 30$ & $04: 54-$ & $y$ & $y \quad a / b$ & \\
\hline 12. 2000-03-14 & S37W90+ & filament & $02: 20-03: 15$ & $02: 26-, 03: 26-$ & $y$ & $n \quad b$ & \\
\hline 13. $2000-03-17$ & S13E90+ & [AR8919?] & $00: 25-01: 45$ & $01: 28-$ & $y$ & $y \quad b / c$ & \\
\hline 14. $2000-03-18$ & N40E90+ & [AR8920?] & $01: 40-02: 30$ & $21: 08-$ & $y$ & $n \quad b$ & \\
\hline 15 & S08E90+ & [AR8921?] & $01: 40-02: 30$ & 21:08- NE & $y$ & $y \quad b / c$ & \\
\hline 16. $2000-03-18$ & S17E90+ & [AR8921?] & $23: 10-03: 00$ & $23: 55^{-}, 02: 30-$ & $y$ & $y \quad b$ & 01:56-02:02-02:10 C5.5 \\
\hline 17. $2000-05-04$ & S13W90+ & {$[\mathrm{AR} 8977]$} & $04: 35-05: 30$ & $04: 50-$ & $n ?$ & $n \quad b / c$ & 04:32-04:51-05:02 M2.8 \\
\hline 18. $1999-02-08$ & N42E90 & filament & 04:30-06:00 & $05: 32-\mathrm{SE}$ & $y$ & $n$ & \\
\hline 19. 1999-02-08 & N58E90 & filament & $23: 00-01: 40$ & $01: 30-$ & $n$ & $y \quad b / c$ & \\
\hline 20. $1999-02-25$ & N45W90+ & filament & 00:00-03:00 & $18: 26-\mathrm{NP}$ & $y$ & $y \quad c$ & \\
\hline 21. 1999-03-07 & S14W90 & AR8478? & 23:20-00:00 & $01: 26-$ & $y ?$ & $y \quad b / c$ & \\
\hline 22. $1999-03-10$ & S78W16 & filament & $01: 10-02: 50$ & $03: 26-$ & $y$ & $n \quad a$ & \\
\hline 23. 1999-03-13 & S45W90+ & filament & 05:00-06:15\# & $06: 26-$ & $y$ & $y \quad a / b$ & \\
\hline 24. 1999-03-16 & N45W90+ & filament & $23: 20 \#-02: 00$ & $01: 26-$ & $y$ & $y \quad a$ & \\
\hline 25. $1999-03-17$ & N52W90+ & filament & 02:00-06:00 & $01: 26-$ & $y$ & $y$ & \\
\hline 26. 1999-04-22 & S25W90+ & [AR8512?] & 01:20-02:00 & $02: 00-$ & $y$ & $y$ & \\
\hline 27. 1999-04-23 & S30W90+ & [AR8514?] & $01: 26-01: 55$ & $01: 50-$ & $y$ & $n$ & \\
\hline 28. 1999-05-08 & N20E90 & AR8541 & $23: 40-01: 00$ & $00: 26-$ & $y$ & $y \quad a$ & 00:36-00:47-00:58 M1.1 \\
\hline 29. 1999-05-14 & S21W67 & filament & $04: 20-06: 30$ & $06: 50-$ & $y$ & $y \quad a / b$ & \\
\hline 30. 1999-05-16 & N43W90+ & filament & $23: 20-02: 30$ & $00: 50-$ & $n$ & $y \quad c$ & \\
\hline 31. 1999-05-24 & S04W90 & QR & $23: 00-01: 00$ & $01: 27-$ & $y$ & $y \quad a / b$ & \\
\hline 32. 1999-06-04 & N18W75 & AR8552 & $05: 30-06: 10$ & $07: 26-$ & $y$ & $n \quad b$ & \\
\hline 33. 1999-06-16 & N34E90 & QR? & $03: 20-04: 40$ & $23: 54-, 04: 54-$ & $y$ & $n \quad b$ & \\
\hline 34. $1999-07-16$ & N40E90+ & [AR8636?] & $00: 30-01: 45$ & $00: 40-$ & $y$ & $n \quad a / b$ & \\
\hline 35. 1999-07-22 & S27E90+ & {$[\mathrm{AR} 8644]$} & $23: 30-00: 30$ & $00: 06-$ & $y$ & $y \quad b$ & \\
\hline 36. 1999-07-27 & N35E90+ & {$[\mathrm{AR} 8651]$} & $00: 30-02: 30$ & $01: 31-$ & $y$ & $y$ & 00:58-02:54-04:51 C2.1 \\
\hline 37. 1999-08-05 & S38W61 & AR8645 & $23: 50-01: 15$ & $23: 26-$ & $y$ & $y \quad b$ & 00:03-00:08-00:12 C3.9 \\
\hline 38. 1999-08-11 & N27W90+ & [AR8651] & $22: 30 \#-23: 40$ & $22: 30-$ & $n$ & $y \quad b / c$ & 22:00-22:29-23:04 C1.5 \\
\hline 39. & N36W90+ & [AR8651] & $23: 40-00: 50$ & $00: 30-$ & $n$ & $y \quad b / c$ & 00:00-00:25-01:15 C1.8 \\
\hline 40. 1999-09-02 & S36W17 & AR8679 & $23: 55 \#-00: 16$ & 00:06- SP & $n$ & $n \quad b / c$ & $23: 30-\mathrm{C} 1 . ?$ \\
\hline 41. 1999-09-14 & N44W90 & filament & 05:00-06:15\# & $06: 30-$ & $y$ & $y ? \quad a$ & \\
\hline 42. $1999-09-21$ & N22W89 & AR8699 & 03:00-03:45 & $03: 30-$ & $n$ & $n b / c$ & 03:00-03:32-03:41 C5.2 \\
\hline 43. $1999-10-20$ & S09E89 & AR8739 & $01: 05-01: 50$ & $23: 26-$ & $y$ & $n \quad a$ & \\
\hline 44. $1999-10-23$ & N50W90+ & filament & 01:00-03:30 & 01:26- NW+SW & $y$ & $n$ & \\
\hline 45. 1999-11-06 & S09E55 & AR8754 & $23: 32-23: 52$ & $23: 38-$ & $y$ & $n \quad a$ & \\
\hline 46. $1999-11-28$ & S69W90+ & filament & 00:30-03:00 & $02: 30-$ & $y$ & $y$ & \\
\hline 47. $1999-12-16$ & N25E90+ & [AR8806] & $03: 15-04: 15$ & - & $y$ & $-\quad-$ & \\
\hline 48. & N25E90+ & [AR8806] & 05:00-06:00\# & - & $y$ & $-\quad-$ & \\
\hline 49. $1999-12-28$ & N38W47 & AR8806 & $01: 00-02: 10$ & $00: 54-$ & $y$ & $y$ & 00:39-00:48-00:52 M4.5, Type II \\
\hline 50. $1999-12-31$ & N80E90 & QR & $23: 00 \#-05: 00$ & $02: 30$ & $y$ & $y$ & \\
\hline
\end{tabular}

a Active regions behind the limb are given in square brackets.

b Time(s) in UT when the CME(s) associated with the prominence eruption, which was verified with the $I-I_{0}$ map, initially appeared in the sequence of $\mathrm{C} 2$ images.

c The start-peak-end times in UT for the GOES soft X-ray flares.

I. $\mathrm{y} / \mathrm{n}$ : the prominence moved along the preexisting nearby streamers.

II. $\mathrm{y} / \mathrm{n}$ : continuity between the prominence and the densest portion of the associated CME.

III. Categories of the mass trajectories $a, b$, and $c$ (see Fig. 11 for definition); $a: 17.0 \%, b: 46.8 \%, c: 4.3 \%$.

$a / b$ means $a$ and/or $b(10.6 \%)$ and $b / c$ means $b$ and/or $c(21.3 \%)$. 
is seen as a dark filament whilst appearing bright above the limb against the dark sky, just similar to those in $\mathrm{H} \alpha$. Note that, the brightness distribution of erupting prominences is slightly different between $\mathrm{H} \alpha$ and $17 \mathrm{GHz}$ images, which is mainly because NoRH observations are not affected by Doppler effects (Hanaoka et al. 1994). Prior to, or at the onset of the eruption, the dark filament can often get heated up and becomes indistinguishable against the quiet Sun as its $T_{\mathrm{b}}$ reaches to $10^{4} \mathrm{~K}$ (Hanaoka \& Shinkawa 1999). It is thus often difficult to identify the initial location of the eruptive filament unless it is heated to $>10^{4} \mathrm{~K}$.

We surveyed $17 \mathrm{GHz}$ prominence eruptions from NoRH daily observations near solar maximum, between February 1999 and May 2000. We initially picked up all eruptions that were observed outside of the solar limb so that their vertical relationship with other coronal structures can be well determined. Smaller or collimated mass ejections, whose counterparts in $\mathrm{H} \alpha$ can be sprays and surges, are also included in this survey. We then selected 50 events that had observations from the beginning of the eruption with NoRH, SOHO LASCO C2, and Yohkoh soft X-ray telescope (SXT; Tsuneta et al. 1991), as listed in Table 1. The latter requirement enables us to find the soft $\mathrm{X}$-ray high temperature $\left(10^{6-7} \mathrm{~K}\right)$ signatures of the eruption in the lower corona, as well as the extent of the coronal activity in white-light. We used daily $\mathrm{H} \alpha$ full-disk images from Big Bear Solar Observatory (BBSO) to identify the location of dark filaments. Radio dynamic spectra from HiRAS (Hiraiso Radio Spectrograph, Communications Research Laboratory, Japan) were also used to examine the existence of metric radio bursts associated with the prominence eruptions.

The LASCO C2 coronagraph observes the outer corona within 2.0-6.0 $R \mathrm{~s}$ (where $R \mathrm{~s}$ is the solar radius, $\sim 7 \times$ $10^{10} \mathrm{~cm}$ ), with a typical cadence of 10-60 min for fullresolution modes $(1024 \times 1024$ pixel size, where one pixel corresponds to 11.9 arcsec). We made white-light synoptic maps for individual events using all of the $\mathrm{C} 2$ full-resolution images available (processing level-0.5) by taking a slice of each image at a specified heliocentric distance above both east and west, or northern and southern limbs. Because the large-scale coronal morphology becomes highly complicated toward the solar maximum (e.g., Saito et al. 1993; Wang et al. 2000), we focus on the corona relatively close to the Sun so that the solar disturbances observed at different heights can be identified. We select three heliocentric distances of $2.5,3.5$, and $4.5 \mathrm{R}$ s for the synoptic maps with a latitudinal resolution of $0.28,0.20$, and 0.16 degree per pixel, respectively (Fig. 1). Intensity at a pixel within a slice is the value averaged over three adjacent pixels located at the same heliographic latitude. For a comparison with NoRH images that were synthesized with less than 10 min cadence, we performed a spline fit of the cubic function to the original slices along the time axis and then obtained synoptic maps that are consisted of $40 \mathrm{~s}$ time slices. We call this modified map "I map", in which the preexisting quiescent structure, or streamer, will appear as a uniform band

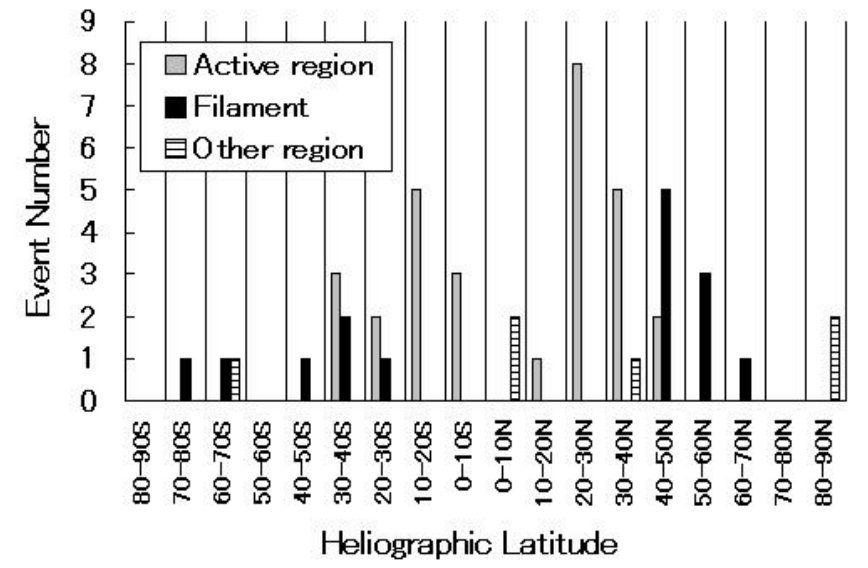

Fig. 3. Latitudinal distribution of the 50 eruptive prominences in intervals of 10 degree.

(Fig. 2a). If this structure has not received any perturbation throughout the event, the band will maintain its (angular) width and pattern. In each $I$ map the initial slice, which we call " $I_{0}$ ", was taken from the $\mathrm{C} 2$ map that was obtained before the start time of the prominence activity. Next, by subtracting the intensity of $I_{0}$ from the whole $I$ map, we made a differential synoptic map, " $I-I_{0}$ map", where the majority of preexisting quiescent structures are subtracted and thus only coronal transients are expected to appear (Fig. 2b). CMEs are distinguished by significant changes in the $I-I_{0}$ map. We marked the heliographic latitude of the top of the activated prominences as a function of time onto the set of $I$ and $I-I_{0}$ maps (the line of black asterisks in Fig. 2). Note the difference in the height between the microwave prominence and the whitelight coronal transients. Time increases from the left to the right in the constructed maps, which is opposite to a typical synoptic map. In order to obtain an overview of the long-term temporal variation of coronal streamers, we also used LASCO C2 Carrington maps constructed at $2.5 R \mathrm{~s}$ for one solar rotation (27.3 days) by the Naval Research Laboratory.

All of the features studied here are 2-D projections in the plane of the sky, which can cause an uncertainty in the interpretation of spatial and causal relationship between the features. This is true especially for emission distant from the plane of the sky (Hundhausen 1993). However, the $I-I_{0}$ map can be a useful tool to find out the latitudinal distribution of coronal transients more clearly than with the previous synoptic maps since emission from preexisting quiescent features has been subtracted.

\section{Results}

\subsection{Statistical properties of the eruptive prominences}

In addition to the actual rising period of the prominences, we defined the duration of the individual prominence activity as the period during which a gradual growth in shape and/or some systematic motions were found in a sequence of NoRH prominences images. (See Table 1 for 


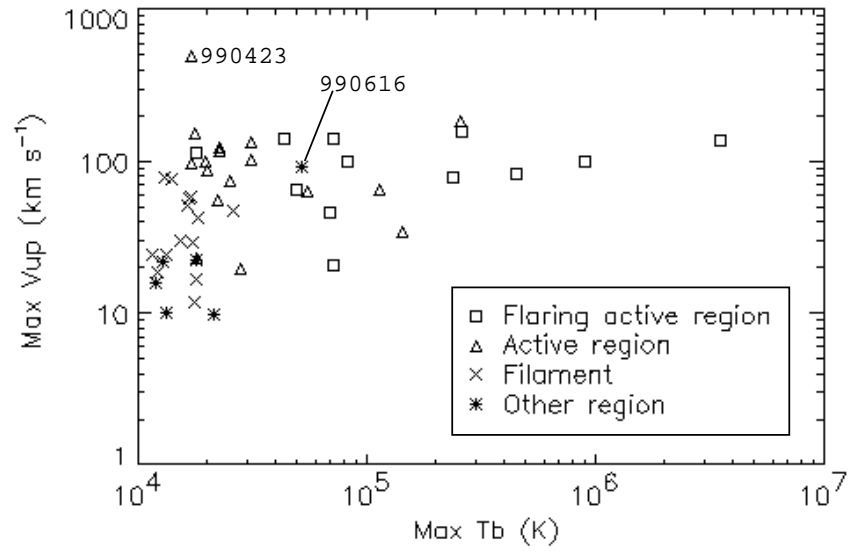

Fig. 4. The maximum brightness temperature $\left(T_{\mathrm{b}}\right)$ vs. the maximum eruption speed $\left(V_{\mathrm{up}}\right)$ of the 50 prominences.

Table 2. Averaged parameters for the 50 prominences.

\begin{tabular}{lcc}
\hline Initial location & $\operatorname{Max} T_{\mathrm{b}}[\mathrm{K}]$ & $\operatorname{Max} V_{\mathrm{up}}\left[\mathrm{km} \mathrm{s}^{-1}\right]$ \\
\hline Flaring active regions & $4.9 \times 10^{5}$ & 99. \\
Active regions & $5.1 \times 10^{4}$ & 114. \\
Filaments & $1.6 \times 10^{4}$ & 40. \\
Other Regions & $2.2 \times 10^{4}$ & 28. \\
\hline
\end{tabular}

the start and end time of the prominence activity for each event.) We obtained an average duration of $1.5 \mathrm{~h}$ for 41 events, excluding 9 events that started before, or continued after, the NoRH observation window (from 23:00 to 7:00 UT). The shortest duration is $20 \mathrm{~min}$ and the longest is $>6 \mathrm{~h} 40 \mathrm{~min}$.

On the basis of the comparison of $17 \mathrm{GHz}(\mathrm{NoRH})$, soft X-ray (SXT), and $\mathrm{H} \alpha$ (BBSO) full-disk images and NOAA active region maps, we inferred the initial location of the 50 eruptive prominences and then categorized them into following three groups: i) active regions with NOAA number (29 events), ii) parts of $\mathrm{H} \alpha$ dark filaments (15 events), and iii) other regions that could not be specified to category i) and ii); such as quiet regions without $\mathrm{H} \alpha$ filaments (6 events). Figure 3 shows the distribution of the initial heliographic latitudes of the prominences for the three groups. In 38 out of the 50 events, the prominence eruptions were initially found at the east or west limb (i.e., W90 or E90 in Table 1). However, this does not necessarily mean that the eruption started exactly at the limb. As mentioned in Sect. 2, prominences passing over the disk with $T_{\mathrm{b}} \sim 10^{4} \mathrm{~K}$ will abruptly appear when they reach the limb. Prominences that were launched behind the limb will also suddenly appear from the limb. For each event, we checked these possibilities by comparing the time evolution of the maximum $T_{\mathrm{b}}$ in the eruptive prominence with that of major active regions on the disk. In 14 out of the 28 active-region-origin events (category i), the active regions were located behind the limb, which later appeared on, or used to be on the visible surface of the Sun. Their inferred NOAA numbers are given in Table 1 within square brackets. Excluding two events (Nos. 14 and 15), these behind-the-limb active regions were located so close to the limb that the tops of the prominences were already seen in microwaves before the start time of the events. Similarly, in 9 out of the 15 filament-origin events (category ii), the prominences originated in the behind-the-limb parts of the $\mathrm{H} \alpha$ filaments.

Of the 50 prominence eruptions studied, 11 events occurred within flare sites ( 6 on the disk and 5 behind the limb) that were classified as $\geq \mathrm{B}$-class events in GOES 1-8 A flux-time profiles. However, the association with flare activity will increase if we consider the undetected behind-the-limb flares. Figure 4 shows the maximum eruption speed $\left(V_{\mathrm{up}}\right)$ plotted against the maximum $T_{\mathrm{b}}$ at $17 \mathrm{GHz}$ for the 50 prominences. The maximum $V_{\text {up }}$ was deduced from the linear-fitting of the prominence heights projected on the plane of the sky, which we defined as the location of the top of the ascending prominence with $T_{\mathrm{b}}=1.5-2 \times 10^{3} \mathrm{~K}(15-20 \%$ of the disk brightness $)$. An upward acceleration of $\geq 0.001 \mathrm{~km} \mathrm{~s}^{-2}$ was found in 6 events. For a few events that show an upward acceleration (but at most $0.012 \mathrm{~km} \mathrm{~s}^{-2}$ ), we estimated the maximum $V_{\text {up }}$ from the steepest slope in the height plot, i.e., just before the prominence left the NoRH field of view $(\leq 0.7 R \mathrm{~s})^{1}$. In Table 2, the averaged maximum $V_{\mathrm{up}}$ and $T_{\mathrm{b}}$ are given for the prominences originating from the flaring active regions, non-flaring active regions, dark filaments (category ii), and other regions (category iii). Both maximum $V_{\mathrm{up}}$ and maximum $T_{\mathrm{b}}$ tend to be high in the active region events, especially in the flare-associated events but low in the filament-origin events. It is thus possible that the fast eruption $\left(91 \mathrm{~km} \mathrm{~s}^{-1}\right)$ plotted in Fig. 4 as category iii (the 16 June 1999 event) may have originated from an active region. It is also suggestive that the active region that produced the fastest prominence eruption $\left(499 \mathrm{~km} \mathrm{~s}^{-1}\right)$ from behind the limb (the 23 April 1999 event) may also have produced an undetected flare. Note that the maximum $V_{\text {up }}$ is well below the gravitational escape speed $\left(\sim 500 \mathrm{~km} \mathrm{~s}^{-1}\right)$ for majority of the events.

We mention that metric type II radio bursts were detected in only 2 events: one is the M4.5 flare event on the disk $\left(V_{\mathrm{up}}=138 \mathrm{~km} \mathrm{~s}^{-1}\right)$ and the other is non-flare event at the NE limb $\left(V_{\text {up }}=24 \mathrm{~km} \mathrm{~s}^{-1}\right)$. The latter event was also accompanied by a type IV burst. As metric type II bursts are known to be associated with flares with highspeed $\left(\geq 400 \mathrm{~km} \mathrm{~s}^{-1}\right)$ mass ejections or blast waves (e.g., Gosling et al. 1976; Gopalswamy et al. 1997), it appeared that our prominence eruptions can be biased to rather weak disturbances appearing in the lower corona.

\subsection{Association with the coronal structures}

From the set of $I$ and $I-I_{0}$ maps, we confirm that preexisting streamers play an important role in activating the prominences. In 41 out of the 50 events, prominences were

\footnotetext{
1 The average acceleration of 18 eruptive $\mathrm{H} \alpha$ filaments reported by Gilbert et al. (2000) is $0.086 \mathrm{~km} \mathrm{~s}^{-2}$.
} 


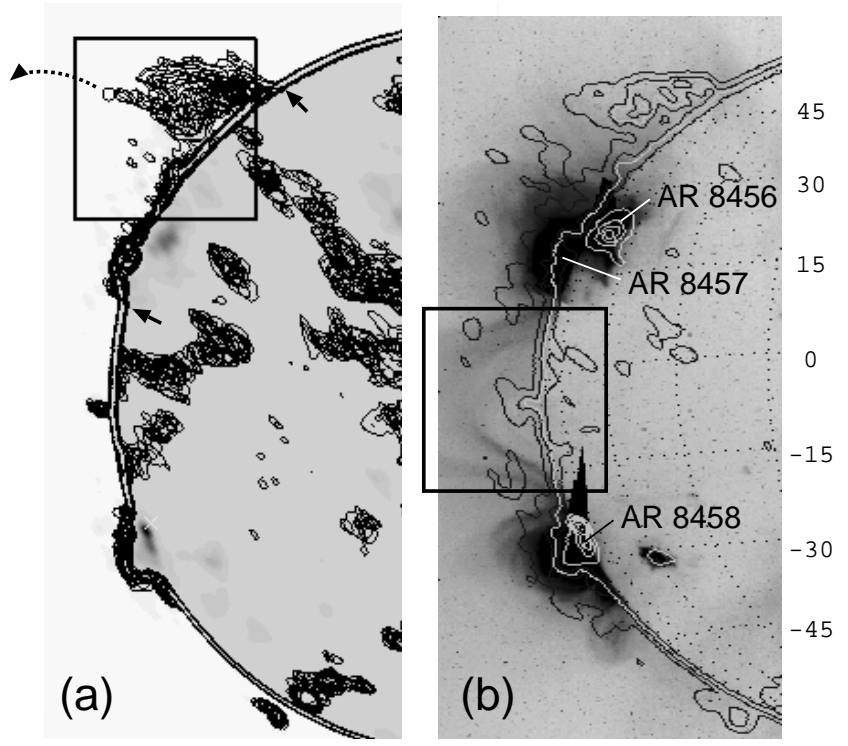

Fig. 5. The 8 February 1999 event. a) NoRH $17 \mathrm{GHz}$ contour maps at $T_{\mathrm{b}}=[0.5,1] \times 10^{4}(\mathrm{~K})$ from 04:50 to 05:46 UT (4 min interval) overlaid on $17 \mathrm{GHz}$ snapshot (04:50 UT). b) SXT snapshot at 04:55 UT (Al Mg filter). $17 \mathrm{GHz}$ contour maps at $T_{\mathrm{b}}=[0.5,1.5,2.0,2.5,3.5] \times 10^{4} \mathrm{~K}$ (white) and $T_{\mathrm{b}}=[0.1$, 1] $\times 10^{4}(\mathrm{~K})$ (black) at 05:08 UT are overlaid. Solar north is up, and east is to the left.

activated within preexisting streamers and then ascended along the streamers (marked "y" in Col. I of Table 1). The remaining 9 events started outside of preexisting streamers and then apparently moved toward nearby streamers. The two helical prominences reported by Hori (2000) belong to the latter group. In spite of the weakness as the lower coronal disturbances, we found that the majority of the events studied ( $92 \%$; 46 events) were associated with CMEs (Table 1 ). In 28 out of the 46 events, the trajectory of the erupted prominence was connected to the brightest portion (or core) of the associated CME, which was verified with the $I-I_{0}$ map constructed at 2.5 Rs (marked "y" in Col. II of Table 1). In the other 18 events, prominence and dense CME material moved along different streamers, i.e., the prominence did not become the CME core. Even for the 4 non-CME events, there was a weak change in the intensity of preexisting streamers along which the prominences ascended, suggesting a weak mass motion.

We describe below four CME-associated events as typical examples in which streamers act as conduits for coronal mass flows.

\subsubsection{The 27 July 1999 event}

This is a helical loop-like prominence, which was initially lying over an active region just beyond the NE limb (AR 8651). The prominence activity started as a compact brightening at its southern footpoint, and then the eruption started at 00:30 UT (Fig. 1). A bright semi-circular loop appeared at the northern end of the AR 8651, to

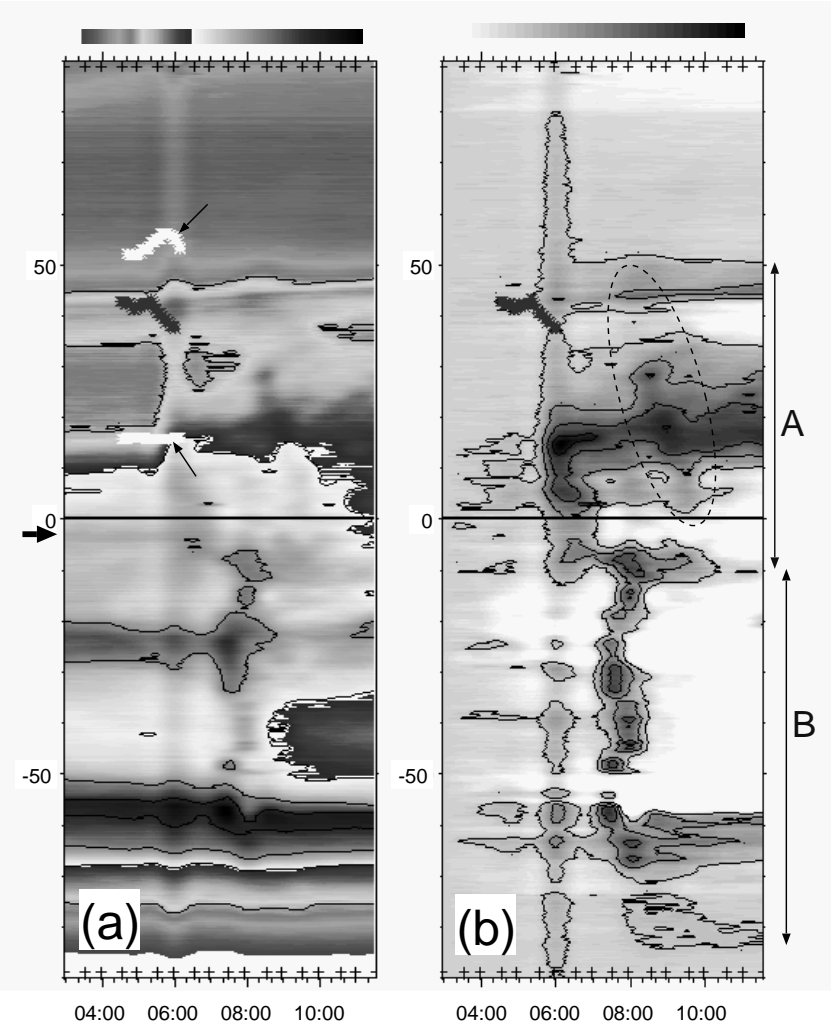

Fig. 6. East limb white-light synoptic maps at $2.5 R$ s for the 8 February 1999 event. a) $I$ map and b) $I-I_{0}$ map. The arrows $\mathrm{A}$ and $\mathrm{B}$ show the latitudinal span of two successive CMEs. The basic format is the same as in Fig. 2.

which the northern leg of the rising prominence attached (01:40 UT). Following the prominence eruption, a gradual C2.1 flare occurred at the AR 8651.

In Fig. 2a, a pair of lines of white asterisks shows latitudes of the northern- and southern-ends of the AR 8651. Before 01:00 UT, a thick uniform band is sandwiched in between the two lines (see black contours), suggesting an existence of a streamer above the AR 8651. The prominence top (the line of black asterisks) initially ascended along the northern edge of the streamer, which gradually deflected northward and then connected to the CME core around $50^{\circ} \mathrm{N}$ (see white arrow). By comparing Figs. 2a and $b$, it is clear that the CME mass was swept away in parallel to a set of preexisting streamers, spanning from $20^{\circ} \mathrm{S}$ to $80^{\circ} \mathrm{N}$. After the passage of the CME front at $2.5 R \mathrm{~s}(02: 00 \mathrm{UT})$, the dense streamer along which the prominence arose disappeared (Fig. 2a) but its outline can be seen as a trace of mass flows afterward (Fig. 2b).

\subsubsection{The 8 February 1999 event}

In this event, a part of a polar crown filament erupted at the NE limb (Fig. 5a). No GOES flare was reported during this event, while the nearest active region (AR 8456 at N23E65; Fig. 5b) showed a gradual $T_{\mathrm{b}}$ increase following the eruption. The northern- and southern-ends of 


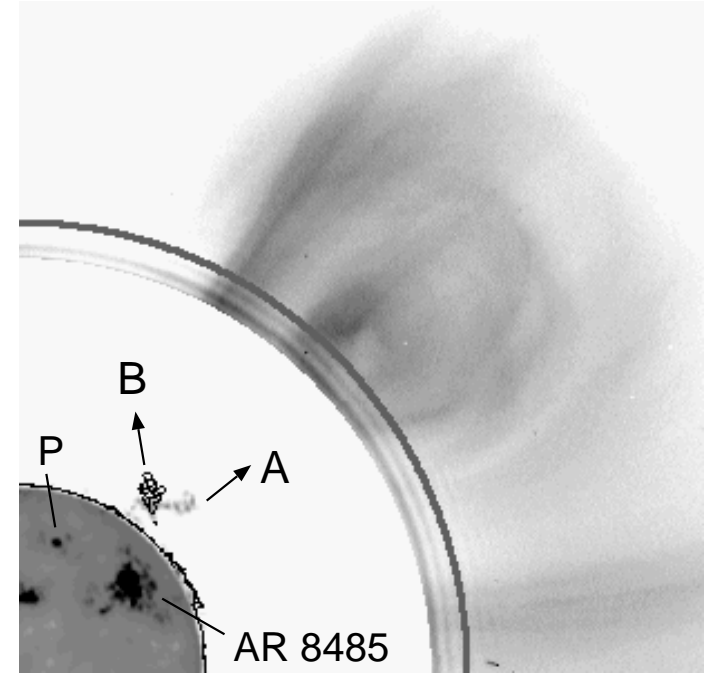

Fig. 7. The 16-17 March 1999 event. NoRH 17 GHz (inner, 01:51 UT) and LASCO C2 (outer, 06:54 UT) image. $17 \mathrm{GHz}$ contours at $T_{\mathrm{b}}=0.4 \times 10^{4} \mathrm{~K}$ are overlaid on the inner image (03:01, 03:31, and 04:01 UT). The arrows A and B indicate the direction of the initial and the second prominence eruptions, respectively. The circle shows the height at $2.5 R$ s. Solar north is up, and west is to the right.

the prominence structure, indicated by arrows in Fig. 5a, are plotted in Fig. 6a with a pair of lines of white asterisks (see thin arrows). The prominence eruption started around 04:30 UT along a faint, thin streamer emanating at $40^{\circ} \mathrm{N}$ (see the line of black asterisks in Fig. 6). As indicated by the dotted arrow in Fig. 5a, the erupting prominence slightly changed its direction southward but remained within the streamer and did not disrupt it. Following the prominence eruption, two CMEs occurred successively from 05:32 UT and 07:00 UT, spanning $50^{\circ} \mathrm{N}$ through $10^{\circ} \mathrm{S}(\mathrm{A})$ and $10^{\circ} \mathrm{S}$ through $80^{\circ} \mathrm{S}(\mathrm{B})$, respectively (Fig. 6b). In spite of the temporal and latitudinal discontinuities, the second CME seems to have affected on mass flows in the preceding $\mathrm{CME}$ (see dotted circle in Fig. 6b). The thin streamer, along which the prominence arose, formed the northern boundary of the CME spreads. The erupted prominence did not merge into the dense portion of either CME.

At the east limb, there is a trans-equatorial soft X-ray loop between AR 8457 (N18E90; beyond the NE limb) and AR 8458 (S23E86) within the box in Fig. 5b. The relationship between this trans-equatorial loop and the two CMEs is not clear: while increasing its height by about $1.5 \times 10^{4} \mathrm{~km}$, the trans-equatorial loop still existed after the CMEs. In microwaves there is a small mound just under the middle of the trans-equatorial loop $\left(5^{\circ} \mathrm{S}\right)$ (Fig. 5), from which a dense streamer emanated in whitelight (thick arrow in Fig. 6a). Since the trajectories of the two successive CMEs are separated around this latitude (Fig. 6b), it is possible that this dense streamer screened the first CME from spanning southwards. This streamer was disrupted after the passage of the second CME front.
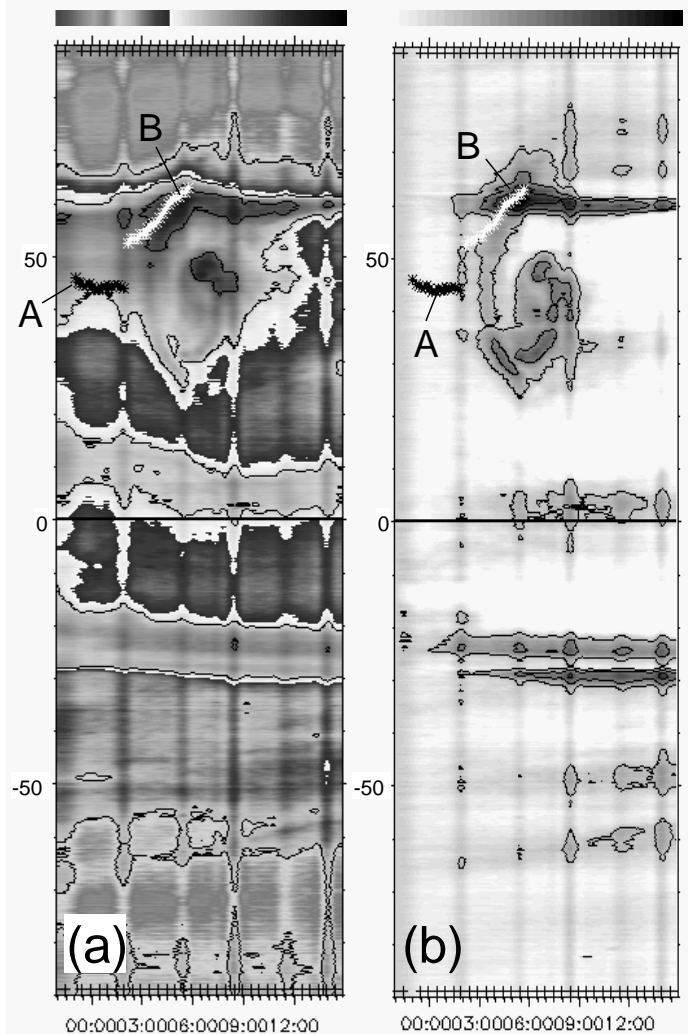

$00: 0003: 0006: 0009: 0012: 00$

Fig. 8. West limb white-light synoptic maps at $2.5 R$ s for the 16-17 March 1999 event. a) $I$ map and b) $I-I_{0}$ map. A line of black asterisks (A) show the latitude of the top of the initial prominence eruption and a line of white asterisks (B) show that of the second eruption. The basic format is the same as in Fig. 2.

\subsubsection{The 16-17 March 1999 events}

These are two prominence eruptions that progressively occurred at the same site but towards different directions (Fig. 7). These prominences were initially located at the NW limb, apparently within a dense streamer with an angular width of $\sim 15^{\circ}$ (Fig. 8a). Following the first prominence eruption (A), a CME with a circular-pattern emerged from the swelling streamer. In Fig. 8, it is clear that the trajectory of the eruption (A) traced the southern edge of the thick streamer (the line of black asterisks), while that of the second eruption (B) just coincides with the northern edge of the circulating CME core (the line of white asterisks). In spite of the difference in height between the prominence ( $\leq 1.7 R$ s from the Sun center) and the CME $(2.5 R \mathrm{~s})$, this coincidence implies that the CME core, or the magnetic field line that involved in the CME, is still connected to the solar surface, at least until 05:41 UT when the prominence entirely faded away.

During this event, impulsive C-class flares occurred three times at the nearest AR 8485, but the relation with the prominence behavior is not clear. On the other hand, a small active region near the prominence site $(\mathrm{P})$ brightened $\left(T_{\mathrm{b}}=4.5 \times 10^{4} \mathrm{~K}\right)$ when the prominence $(\mathrm{A})$ left 


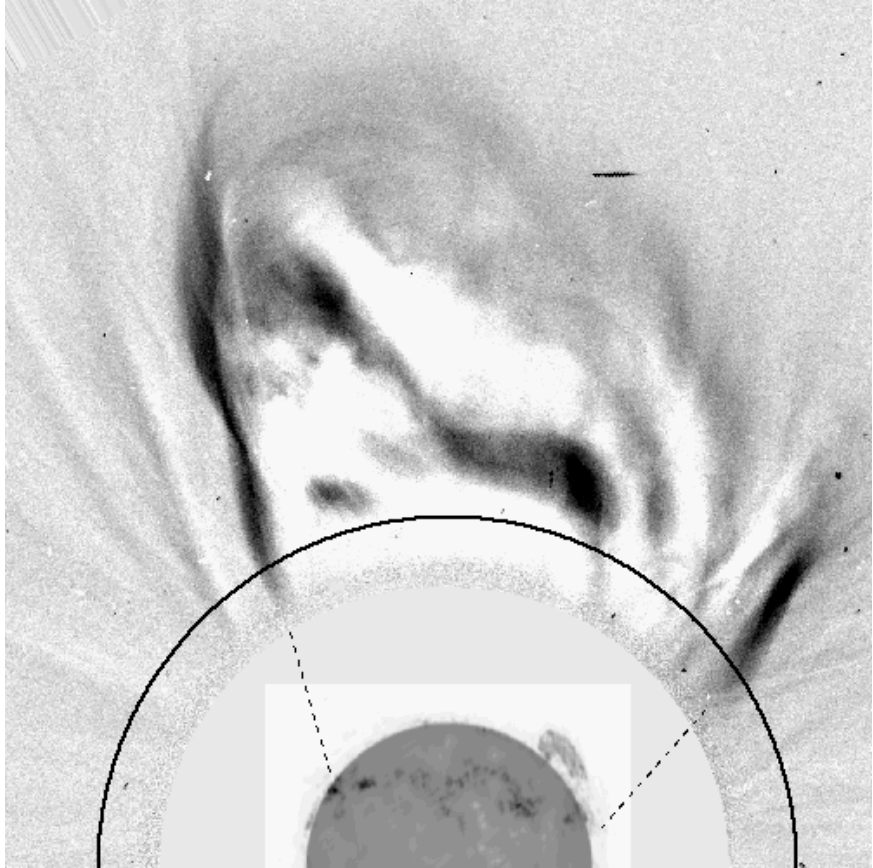

Fig. 9. The 25 February 1999 event. NoRH $17 \mathrm{GHz}$ image (inner, 01:31 UT) and LASCO C2 running difference image (outer, 01:26-00:54 UT). Note that the CME was launched from the North polar region around 18:30 UT on the 24th. The circle shows the height at $2.5 R \mathrm{~s}$. Solar north is up.

the NoRH field of view, indicating a magnetic connection with the prominence.

\subsubsection{The 25 February 1999 event}

This is an expanding large prominence arch, which was initially lying at the NW limb. Prior to the prominence eruption, a faint CME was launched from the north polar region around 18:30 UT on February 24 (Fig. 9). Neither microwave nor soft X-ray loops can be seen around the north polar region. Instead, a long, winding belt lies over the northern hemisphere around $30^{\circ} \mathrm{N}$. This belt consists of groups of soft X-ray loops seen by SXT, which also can be seen as a bright belt at $17 \mathrm{GHz}$ (Fig. 9, where higher intensity is darker) and as a bright plage in $\mathrm{H} \alpha$. A polar crown filament overlies the western half of the plage belt.

This CME initially arose from a low density region surrounded by preexisting streamers (arrow in Fig. 10b) and finally developed into a huge, helical-cone structure bounded by the streamers. Within the east- and west-side streamers, which were pushed aside toward lower latitudes in response to the CME as indicated by arrows in Fig. 10a, dense coronal material flowed out almost simultaneously. The prominence eruption started around 00:00 UT following these large-scale coronal disturbances. A pair of lines of white asterisks in Fig. 10a marks the latitude of the northern- and southern-legs of the prominence, while the line of black asterisks in Figs. 10a and b marks that of the prominence top. The huge prominence ascended along the west-side streamer and then merged into the densest
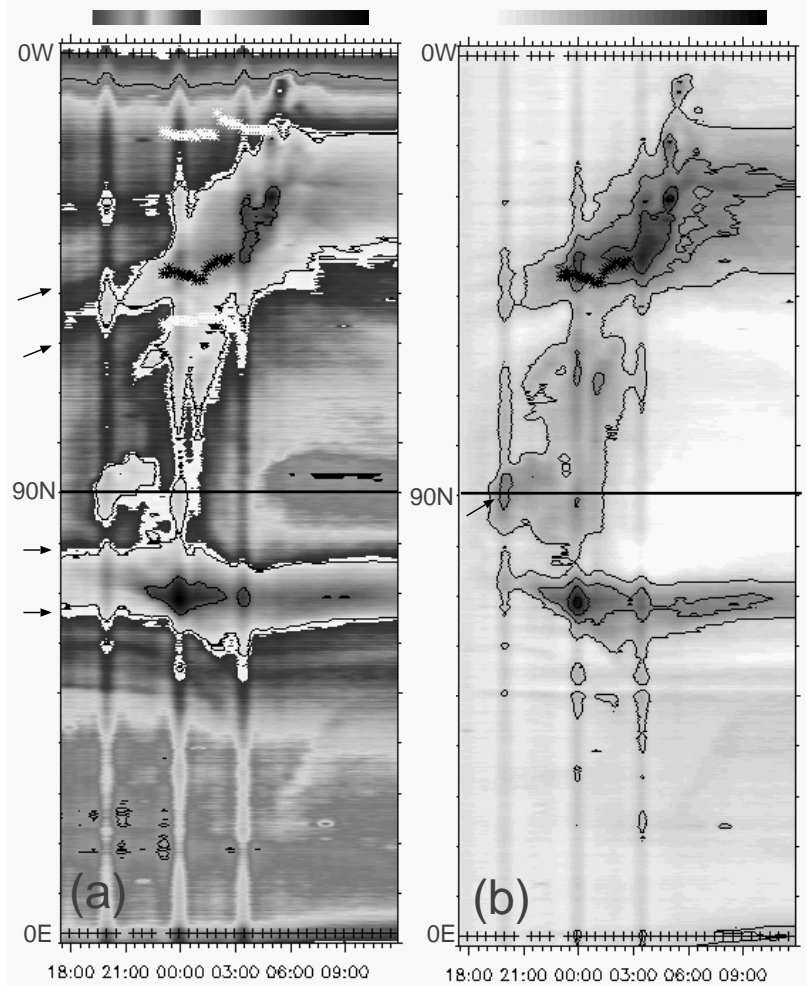

Fig. 10. North polar limb white-light synoptic maps at $2.5 R \mathrm{~s}$ for the 25 February 1999 event. a) $I$ map. The arrows indicate the latitudes of preexisting streamers. b) $I-I_{0}$ map. The basic format is the same as in Fig. 2.

portion of the CME. After 04:30 UT, a large soft X-ray structure appeared beyond the NW limb, where the huge prominence had existed. There is no report of GOES soft $\mathrm{X}$-ray flares during this event. Note that the angular width of the helical-cone CME almost coincides with the latitudes of the long winding belt on the solar surface (see dashed lines in Fig. 9).

\section{Summary and discussion}

On the basis of a survey of microwave prominence eruptions using NoRH $17 \mathrm{GHz}$ images near solar maximum (1999-2000), we examined the relationship between the prominence activity and the surrounding coronal structures. This study is consequently weighted towards weak coronal disturbances: of the 50 events studied, only 12 events occurred at $\geq$ B9. 2 flare sites and only 2 events occurred associated with radio types II and IV bursts. However, the association with flare activity will increase if we consider the possible behind-the-limb flares. On the other hand, 46 events had a CME association, which was verified with white-light synoptic maps ( $I$ and $I-$ $I_{0}$ maps) from the LASCO C2 coronagraph. Hundhausen (1993) concluded that most CMEs originate in the bright streamer belts. In this study, we provide further evidence that streamers organize the coronal mass flows, including both eruptive prominences and CMEs. 


\subsection{Trajectories of coronal mass motions}

In spite of the uncertainty due to projection effects, the tops of the eruptive prominences were found to move along preexisting streamers in $82 \%$ of the events. The remaining events started outside of preexisting streamers and apparently moved toward the nearest streamer (not shown in this paper, see Hori 2000). CME material also tends to move in a path parallel to preexisting streamers. Oneto-one correspondence between the erupted prominence and the dense portion of the CMEs could be confirmed in only $61 \%$ of the $46 \mathrm{CME}$-associated events. As reported by Webb et al. (1997), the original position of the eruptive prominence is not necessarily centered under the associated CME. Some prominence eruptions show a systematic latitude offset from the latitudinal span of their associated CMEs (e.g., the 8 February 1999 event). Even in these cases, we found that both eruptions are linked to each other through preexisting streamers.

Since the Skylab era, it has been known that CMEs have a variety of structural shapes (Munro et al. 1979; Wagner 1984; Howard et al. 1985). Howard et al. (1985) discussed what kind of physical properties are reflected in the structural difference of CMEs, e.g., the amount of energy release, the magnetic field configurations in the region of propagation, or some other mechanism. Our study points out the importance of the background magnetic field configuration.

The $46 \mathrm{CME}$ structures identified on the synoptic maps can be divided into three groups with respect to their relation with preexisting streamers (Fig. 11): (a) CMEs that emerged from, and were expelled along, a single streamer (e.g., the 16-17 March 1999 event), (b) CMEs that encompassed multiple streamers (e.g., the 8 February 1999 and the 27 July 1999 events), and (c) CMEs that arose from a sparse mass region outside of, or surrounded by, streamers (e.g., the 25 February 1999 event). In order to clarify the relationship between preexisting streamers and coronal mass motions, we did not sketch the deformations of the streamers by the mass motions in Fig. 11. For the classification, we used all of the synoptic maps constructed at $2.5,3.5$, and $4.5 R$ s to verify the extent of the relation. The results are given in Col. III of Table 1 , as well as the fractional distribution. Group (a) might be included in group (b) since CMEs expelling a single streamer can often weakly affect surrounding streamers (e.g., the 1617 March 1999 event) ${ }^{2}$.

\subsubsection{Multiple sheet corridor}

The bundle of streamers involved with CMEs in group (b) may suggest that each streamer has a sheet structure, i.e., the CME encompasses "a multiple plasma sheet" (Fig. 12, right). Since the 1970s, it has been recognized

\footnotetext{
2 The "streamer blowout" and "spike" CMEs defined in Howard et al. (1985) might correspond to our group (a) while their "double spike" and "multi spike" CMEs would be included in our group (b).
}

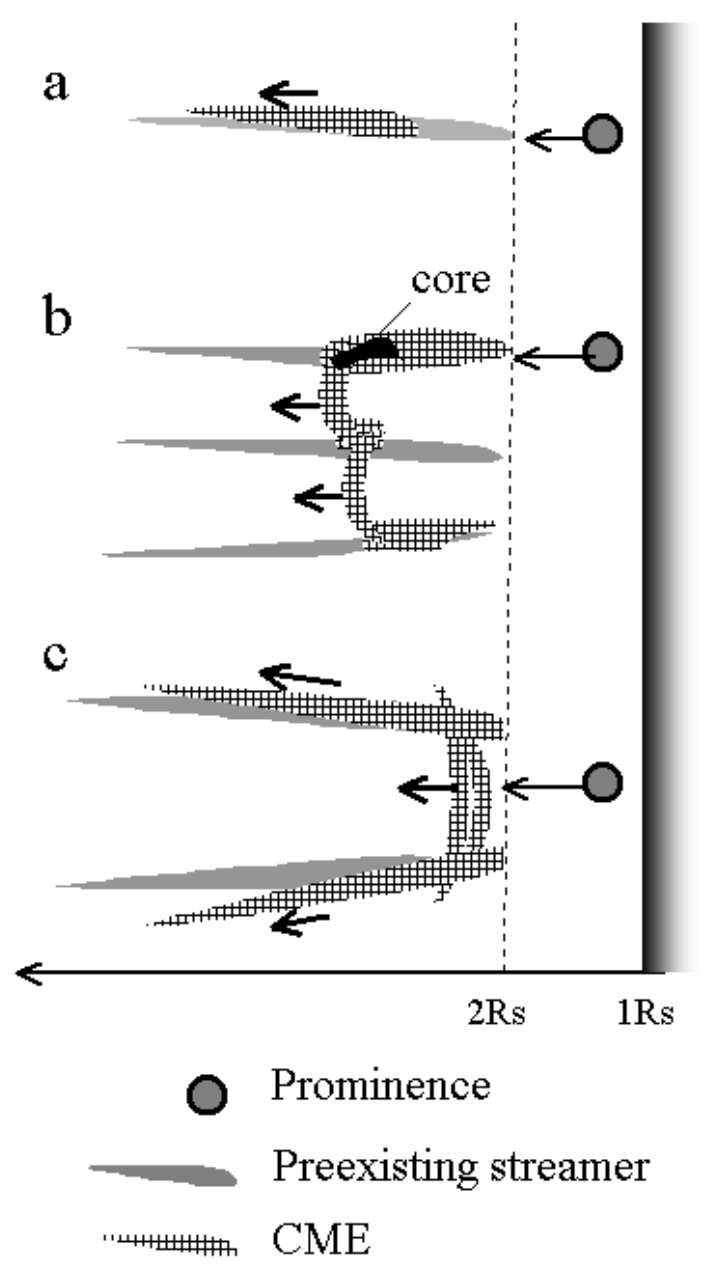

Fig. 11. Schematic diagram of trajectories of the eruptive prominences and CMEs. a) along a single streamer, b) along bundles of streamers, c) outside of, or surrounded by streamers. The solid arrows indicate coronal mass motions including both the eruptive prominences and CMEs. The vertical dashed line indicates the location of the edge of the $\mathrm{C} 2$ occulting disk.

that large-scale coronal streamers can be represented by an edge-on view of a single warped heliospheric current sheet (HCS), encircling the Sun (e.g., Saito et al. 1993; Wang et al. 1997). This single-sheet interpretation, however, seems to fail for the CMEs that progressively occurred within multiple layers of streamers with a discontinuity across one of the streamers (e.g., the 8 February 1999 event); these CME outflows might have been separated by a sheet structure.

Using the set of $I$ maps, we verified that the structure of the bundle of streamers can be maintained at least up to $4.5 \mathrm{Rs}$. While it is possible that these separate structures may finally come into contact at a single HCS far above the solar surface, the multiple layers might still exist as highly structured fields observed at the sector boundary in the heliosphere (Crooker et al. 1993). Crooker et al. replaced the concept of a single, rippled HCS with a bundle of many current sheets that originates from multiple helmet arcades covering active regions and high-latitude 


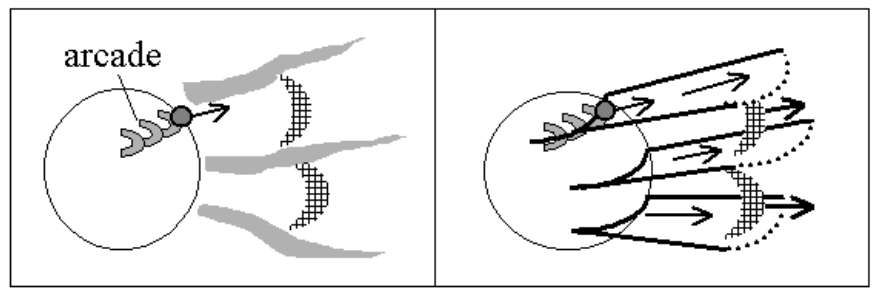

Fig. 12. Multiple sheet corridor. The circle represents the Sun and the arrows indicate the direction of the mass outflows. Left: schematic diagram of CMEs belong to group (b). Right: our interpretation.

filaments. The resemblance between their sketch of the multiple HCS (Fig. 1b in Crooker et al. 1993) and our prominence and CME trajectories categorized (b) may support the idea that coronal mass motions are organized by multiple plasma sheets. We have no evidence of sheet currents, though. In fact, the latitudinal span of CMEs involved with a combined set of streamers appears to have been bounded by active regions and polar crown filaments (dashed lines in Figs. 1 and 9). Hence it seems reasonable that flare activity associated with CMEs tends to lie under the boundaries, rather than the center, of the CME spread (e.g., Harrison et al. 1990; Kahler 1991). Further, we can anticipate that the long-term temporal variation of CME properties would correlate with the solar activity cycle through changes in the distribution of active regions and polar crown filaments (e.g., Hildner et al. 1976; Howard et al. 1986).

\subsubsection{Sparse mass corridor}

On the other hand, CMEs can originate from a low density region surrounded by streamers (group (c); Fig. 13, left). Although we cannot see $3-\mathrm{D}$ structures of coronal mass motions, we propose that CMEs in group (c) are guided by a huge magnetic cone consisting of an extremely warped (outermost) plasma sheets (Fig. 13, right), rather than a huge gap sandwiched by a pair of plasma sheets as seen at low-latitude (Fig. 13, middle). Otherwise, the helical-cone-structured CME bounded by streamers in the 25 February 1999 event will not appear. Considering the occurrence rate of group (c) events, passage of CMEs through such a "sparse mass corridor" seems a rare case.

Our results may contradict the Subramanian et al. (1999) study with LASCO C2 data, in which about $46 \%$ of their 375 CMEs (including Halo CMEs) have no effect on the streamers, even though they appear to overlap, and only $16 \%$ show the disruption of streamers. Using the $I-I_{0}$ maps, we found minor changes in preexisting streamers in association with both prominence and CME material. We found that not all CMEs disrupt the preexisting streamers. Especially, streamers that consist of the outermost layer of the multiple sheets tend to remain connected to the Sun after the passage of CMEs, which was

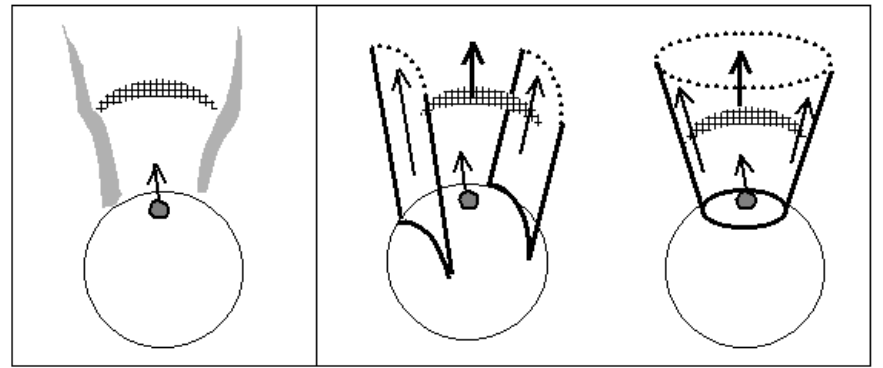

Fig. 13. Sparse mass corridor. Left: schematic diagram of CMEs belong to group (c). Middle and right: our interpretation. A corridor sandwiched by a pair of plasma sheets (middle) and a cone-shaped corridor, or extremely warped (outermost) plasma sheet (right).

verified with the NRL C2 Carrington maps constructed for one solar rotation. This tendency is consistent with Kahler's (1991) report that streamers overlying the highlatitude active regions appeared to develop and evolve independently of the occurrence of CMEs. We point out that most of our events were not associated with flares, while more than $50 \%$ of major CMEs appear be associated with flares (comment from N. Nitta 2001). This might be the main reason for the difference between our results and Subramanian et al.'s results.

\subsection{Base of the streamers}

Although our study may support the multiple HCS model, we could not confirm one-to-one correspondence between location of individual streamers and the magnetic neutral lines that should underlie the streamers (Webb et al. 1997). Instead, we verified that dense streamers tend to emanate from latitudes of active regions and polar crown filaments, suggesting that streamer material basically comes from active regions (Wang et al. 1998; Liewer et al. 2000), soft X-ray arcades (McAlllister \& Hundhausen 1996), and also from trans-equatorial interconnecting loops (e.g., the 8 February 1999 event). We found that even the non-CME events could be associated with weak mass outflows confined to streamers at which base a prominence erupted. These weak outflows might correspond to the white-light plasma blobs reported by Sheeley et al. (1997) and Wang et al. (1998). Therefore, as pointed by Crooker et al. (1993), frequent mass outflows might exist from the base of the streamers, not only during periods of eruptive activity but also during quiet periods.

Since most CMEs studied were sandwiched by preexisting streamers (Fig. 12, left), the latitudinal span of individual CMEs would depend on the distribution of streamers. It is thus possible that CME legs, if they remain connected to the Sun until the late phase of the CME, may correspond to a pair of dense, long-lived streamers. This possibility can be checked by comparing the base of the streamers that produces (or sandwiches) a CME and the magnetic flux rope that might originate from the CME. 
Magnetic helicity (Rust 1994; Rust \& Kumar 1996) and He abundance (Burlaga et al. 1998) are potentially useful parameters to examine their relationship. Even if this conjecture is wrong, a long term observation of streamers will provide useful information on the evolution of CMEs.

\section{Conclusion}

From a combined set of coronal observations in microwave and white-light continuum emission, we have confirmed that coronal mass motions involving eruptive prominences and CMEs are not random but are organized by bundle of streamers. Large scale evolution of coronal features suggests that streamers are a signature of multiple plasma sheets emanating from active regions, arcades, trans-equatorial interconnecting loops, and polar crown filaments, through which coronal mass is transported toward interplanetary space.

Without 3-D observations from the photosphere through the corona, it is difficult to identify the trigger of coronal transients. Most of the prominence eruptions studied here were preceded by low-coronal activities at or near the prominence site, such as flares and the increase of the brightness temperature at $17 \mathrm{GHz}$. However, we found 6 events that started after the appearance of CMEs over 2.0 Rs (e.g., the February 25, 1999 event). As described in Sect. 4.1, all streamers are presumably linked as a part of multiple plasma sheets, encircling the Sun. Hence, pressure pulses induced by, e.g., preceding CMEs may travel around the high corona and finally activate the low corona through streamers (see McComas et al. 1991). This possibility should be compared with other possible causes of coronal transients, especially photospheric activity such as shear motion and emerging flux (e.g., Feynman \& Martin 1995, and references therein).

Our study showed that the ground-based microwave observations can provide useful information on the activity at the base of "coronal mass corridors". For further understanding the creation and propagation of coronal transients, collaborative observations by the future space mission will play a key part. Especially, the combination of Solar-B (Japan/US/UK) and STEREO (US) to be launched in 2005, which are designed for a close view of the Sun's magnetic field and a 3-D study of the Sun-Earth connection, will come up to our expectations.

Acknowledgements. We thank S. Plunkett for information on LASCO observations and N. B. Rich for providing their LASCO C2 Carrington maps. We also thank L. Harra, L. van Driel-Gestelyi, N. Nitta and anonymous referee for helpful comments to improve this paper. The authors greatly benefit from the open data policy of the SOHO, Yohkoh, and Nobeyama Radioheliograph team. Yohkoh/SXT data was provided by the Solar UK Research Facility (SURF) at Mullard Space Science Laboratory. LASCO is a multinational team effort by the Naval Research Laboratory (USA), the Max-Planck Institute für Aeronomie (Germany), the Laboratoire d'Astronomie Spatiale (France), and the
University of Birmingham School of Physics and Astronomy (UK). This work was supported by the Particle Physics and Astronomy Research Council (PPARC).

\section{References}

Brueckner, G. E., Howard, R. A., Koomer, M. J., et al. 1995, Sol. Phys., 162, 357

Burlaga, L., Fitzenreiter, R., Lepping, R., et al. 1998, J. Geophys. Res., 103, 277

Crooker, N. U., Siscoe, G. L., Shodhan, S., et al. 1993, J. Geophys. Res., 98(A6), 9371

Delannee, C., Delaboudiniere, J.-P., \& Lamy, P. 2000, A\&A, 355,725

Fujiki, K. 1999, in Solar Physics with Radio Observations, ed. T. S. Bastian, N. Gopalswamy, \& K. Shibasaki, NRO Rep., 479,249

Feynman, J., \& Martin, S. F. 1995, J. Geophys. Res., 100, 3355

Gilbert, H. R., Holzer, T. E., Burkepile, J. T., \& Hundhausen, A. J. 2000, ApJ, 537, 503

Gopalswamy, N., Kurdu, M. R., Manohavou, P. K., et al. 1997, ApJ, 486, 1036

Gosling, J. T., Hildner, E., MacQueen, R. M., et al. 1976, Sol. Phys., 48, 389

Gosling, J. T., 1997, in Coronal Mass Ejection, ed. N. Crooker, J. A. Joselyn, \& J. Feynman, Geophys. Monograph Ser. 99, AGU, 9

Hanaoka, Y., Kwokawa, H., Enome, S., et al. 1994, PASJ, 46, 205

Hanaoka, Y., \& Shinkawa, T. 1999, ApJ, 510, 466

Harrison, R. A., Hildner, E., Hundhausen, A. J., Sime, D. G., \& Simnett, G. M. 1990, J. Geophys. Res., 95, 917

Hildner, E., Gosling, J. T., MacQueen, R. M., et al. 1976, Sol. Phys., 48, 127

Hori, K. 2000, ApJ, 543, 1011

Howard, R. A., Sheeley, N. R., Jr., Koomen, M. J., \& Michels, D. J. 1985, J. Geophys. Res., 90, 8173

Howard, R. A., Sheeley, N. R., Jr., Michels, D. J., \& Koomen, M. J. 1986, in The Sun and the Heliosphere in Three Dimensions, ed. R. G. Marsden (D. Reidel, Dordrecht), 107

Howard, R. A. 1997, in Coronal Mass Ejection, ed. N. Crooker, J. A. Joselyn, \& J. Feynman, Geophys. Monograph Ser. 99, AGU, 17

Hundhausen, A. J. 1993, J. Geophys. Res., 98, 13177

Illing, R. M. E., \& Hundhausen, A. J. 1985, J. Geophys. Res., 90, 275

Jackson, B. V. 1981, Sol. Phys., 73, 133

Kahler, S. W. 1991, ApJ, 378, 398

Kahler, S. W., Moore, R. L., Kane, S. R., \& Zirin, H. 1988, ApJ, 328, 824

Liewer, P. C., Hall, J. R., DeJong, E. M., et al. 2000, SPD, 32, L1501

Low, B. C. 1994, Phys. Plasmas, 1, 1684

McAlllister, A. H., \& Hundhausen, A. J. 1996, in Solar Drivers of Interplanetary Disturbances, ed. K. S. Balasubramaniam, S. L. Keil, \& R. N. Smartt (Astron. Soc. of the Pac., San Francisco, Calif), 171

McComas, D. J., Phillips, J. L., Hundhausen, A. J., \& Burkepile, J. T. 1991, Geophys. Res. Lett., 18(1), 73 
Munro, R. H., Gosling, J. T., Hildner, E., et al. 1979, Sol. Tsuneta, S., Acton, L., Bruner, M., et al. 1991, Sol. Phys., Phys., 61, 201

Nakajima, H., et al. 1994, Proc. of the IEEE, 82, 705 136,37

Plunkett, S. P., Vourlidas, A., Simberová, S., et al. 2000, Sol.

Wagner, W. J. 1984, ARA\&A, 22, 267

Phys., 194, 371

Rust, D. M. 1994, Geophys. Res. Lett., 21, 241

Rust, D. M., \& Kumar, A. 1996, ApJ, 464, L199

Saito, T., Akasofu, S.-I., Kozuka, Y., Takahashi, T., \& Numazawa, S. 1993, J. Geophys. Res., 98, 5639

Sheeley, N. R., Jr., Bohlin, J. D., Brueckner, G. E., et al. 1975, Sol. Phys., 45, 377

Wang, Y.-M., Sheeley, N. R., Jr., Howard, R. A., et al. 1997, ApJ, 485, 875

Wang, Y.-M., Sheeley, N. R., Jr., Walters, J. H., et al. 1998, ApJ, 498, L165

Wang, Y.-M., Sheeley, N. R., Jr., \& Rich, N. B. 2000, Geophys. Res. Lett., 27, 149

Webb, D. F., \& Hundhausen, A. J. 1987, Sol. Phys., 108, 383

Sheeley, N. R., Jr., Warg, Y.-M., Hawley, S. H., et al. 1997, ApJ, 484, 472

Sime, D. G. 1989, J. Geophys. Res., 94, 151

St. Cyr, O. C., \& Webb, D. F. 1991, Sol. Phys., 136, 379

Subramanian, P., Dere, K. P., Rich, N. B., \& Howard, R. A. 1999, J. Geophys. Res., 104, 22321

Webb, D. F., Kahler, S. W., McIntosh, P. S., \& Klimchuck, J. A. 1997, J. Geophys. Res., 102, 24161

Wiik, J. E., Schmieder, B., Kucera, T., et al. 1997, Sol. Phys., 175,411 